\title{
Nanotechnology as a savior in asthma management
}

\author{
Chin Kook Rhee \\ Division of Pulmonary, Allergy and Critical Care Medicine, Department of Internal Medicine, Seoul St. Mary's Hospital, College of Medicine, The \\ Catholic University of Korea, Seoul, Republic of Korea \\ Correspondence to: Chin Kook Rhee, MD, PhD. Division of Pulmonary, Allergy and Critical Care Medicine, Department of Internal Medicine, \\ College of Medicine, Seoul St Mary's Hospital, The Catholic University of Korea, 222 Banpo-daero, Seocho-gu, Seoul 06591, Republic of Korea. \\ Email: chinkook77@gmail.com. \\ Provenance: This is an invited article commissioned by the Editorial Office, Annals of Translational Medicine. \\ Comment on: Wang L, Feng M, Li Q, et al. Advances in nanotechnology and asthma. Ann Transl Med 2019;7:180.
}

Submitted Sep 05, 2019. Accepted for publication Sep 18, 2019.

doi: $10.21037 /$ atm.2019.09.98

View this article at: http://dx.doi.org/10.21037/atm.2019.09.98

Asthma is common airway disease characterized by airway inflammation. Although treatment outcome has been improved since the use of inhaled corticosteroid (ICS), still there are unmet needs in asthma treatment. Nanotechnology can be a promising tool in asthma management. Nanotechnology can be utilized in the fields of drug delivery and endotype identification. By using this new technology, treatment of asthma can be improved.

Currently, the most important medication in asthma is ICS. Traditionally, asthma has been regarded as eosinophilic inflammatory disease. ICS effectively decreases eosinophilic inflammation and reduces symptom and exacerbation in patients with asthma. However, asthma is a heterogenous disease and there are several phenotypes. In some patients, neutrophilic inflammation is predominant. ICS is not effective on these patients. Currently, there is no recommended treatment for neutrophilic asthma. Recently, anti-IL-5 and other biologics showed promising effect on severe asthma. However, most of drugs currently available target only eosinophilic phenotype. Many biologics including anti-IL-17 failed to show efficacy in neutrophilic asthma patients (1). Nanotechnology can be utilized in the development of new drugs for neutrophilic asthma. There are many potential small molecules that can effectively decrease neutrophilic inflammation. However, due to poor water-solubility, candidate molecule cannot be delivered to the tissue. Nanotechnology can be employed to overcome this problem. By formulating candidate molecule as nanoparticle, deposition of drug can be improved.

ICS treatment should be maintained since asthma is a chronic disease. However, it has been well known that long-term use of ICS can increase the risk of pneumonia, tuberculosis, and nontuberculous mycobacteria (2-4). The risk of side effect was correlated with the dose of ICS. Thus, decreasing the dose of ICS is needed to prevent adverse effects. However, there is a dose dependent response in ICS especially in asthma. Increase of ICS dose is recommended in severe patients. Here is a dilemma of asthma treatment. Nanotechnology can be utilized to decrease the dose of ICS while maintaining its therapeutic effect. Wang et al. reviewed the potential benefits of nanocarrier-based drugs in asthma (5). In this review, how nanotechnology can improve the effect of traditional asthma drugs was elegantly described. Enhancing therapeutic effect while minimizing side effect by nanotechnology also can be applied to other asthma drugs such as bronchodilator.

A treatable trait is defined as a phenotypic or endotypic characteristic that can be successfully targeted with treatment (6). To identify endotype and find treatable traits are extremely important in asthma management. However, still there is lack of good biomarker. Nanotechnology can be used to identify endotype. To find Inflammatory phenotype is essential in precision medicine. However, gold standard for this process is to analyze induced sputum. In clinical practice, it is very difficult to obtain induced sputum. Thus, there has been a challenging to identify underlying inflammatory profile in patients with asthma. Plaza and colleagues utilized nanotechnology to find phenotype of asthma (7). Electronic nose is a novel device consisted of nanosensors that can detect specific volatile 


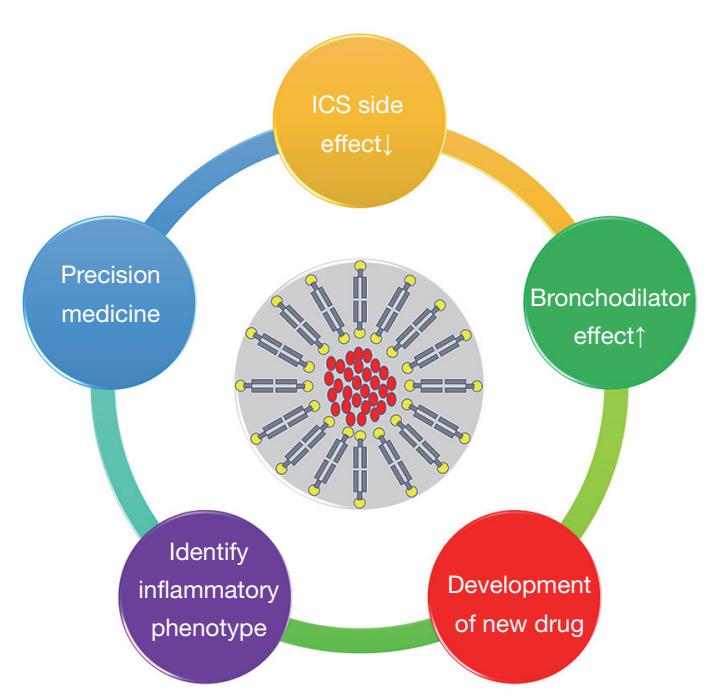

Figure 1 The role of nanotechnology in the management of asthma.

organic compounds (VOCs) in exhaled gas (8). In this study, Inflammatory asthma phenotypes were recognized in induced sputum and VOCs breath-prints were analyzed. An electronic nose can discriminate inflammatory phenotype. The usefulness of electronic nose was comparable to induced sputum. One of the merits of electronic nose is that this technology is noninvasive. It is an easy and simple test that can be readily performed in clinical practice. To classify patients according to inflammatory profile is powerful tool that enable personalized treatment. Nanotechnology, in the future, will be very important and widely used in daily clinical practice.

The nanotechnology is relatively new area in respiratory medicine. However, it has been grown over the last 10 years (8). To apply nanotechnology in asthma is no longer future event. For example, inhaled medication is essential in asthma and dry powder inhaler is most common form. Recently, nano-embedded microparticle (NEM) powder was developed. Keil et al. showed that that spray dried NEM formulations containing nucleic acids can be prepared with characteristics known to be optimal for inhalation therapy (9). Nanotechnology can be used in various ways in the management of asthma (Figure 1). Further prospective clinical trial is needed to validate the effect of nanotechnology in asthma.

\section{Acknowledgments}

None.

\section{Footnote}

Conflicts of Interest: The author has no conflicts of interest to declare.

Ethical Statement: The author is accountable for all aspects of the work in ensuring that questions related to the accuracy or integrity of any part of the work are appropriately investigated and resolved.

\section{References}

1. Busse WW, Holgate S, Kerwin E, et al. Randomized, double-blind, placebo-controlled study of brodalumab, a human anti-IL-17 receptor monoclonal antibody, in moderate to severe asthma. Am J Respir Crit Care Med 2013;188:1294-302.

2. Finney L, Berry M, Singanayagam A, et al. Inhaled corticosteroids and pneumonia in chronic obstructive pulmonary disease. Lancet Respir Med 2014;2:919-32.

3. Lee CH, Kim K, Hyun MK, et al. Use of inhaled corticosteroids and the risk of tuberculosis. Thorax 2013;68:1105-13.

4. Liu VX, Winthrop KL, Lu Y, et al. Association between Inhaled Corticosteroid Use and Pulmonary Nontuberculous Mycobacterial Infection. Ann Am Thorac Soc 2018;15:1169-76.

5. Wang L, Feng M, Li Q, et al. Advances in nanotechnology and asthma. Ann Transl Med 2019;7:180.

6. Agusti A, Bel E, Thomas M, et al. Treatable traits: toward precision medicine of chronic airway diseases. Eur Respir J 2016;47:410-9.

7. Plaza V, Crespo A, Giner J, et al. Inflammatory Asthma Phenotype Discrimination Using an Electronic Nose Breath Analyzer. J Investig Allergol Clin Immunol 2015;25:431-7.

8. Doroudian M, MacLoughlin R, Poynton F, et al. Nanotechnology based therapeutics for lung disease. Thorax 2019;74:965-76.

9. Keil TW, Feldmann DP, Costabile G, et al. Characterization of spray dried powders with nucleic acidcontaining PEI nanoparticles. Eur J Pharm Biopharm 2019;143:61-9.

Cite this article as: Rhee CK. Nanotechnology as a savior in asthma management. Ann Transl Med 2019;7(20):517. doi: 10.21037/atm.2019.09.98 